\title{
Temporal order judgment criteria are affected by synchrony judgment sensitivity
}

\author{
RoB L. J. VAN EIJK \\ Eindhoven University of Technology, Eindhoven, The Netherlands
}

Armin KohlRausch

Eindhoven University of Technology, Eindhoven, The Netherlands and Philips Research Laboratories Eindhoven, Eindhoven, The Netherlands

JAMES F. JUOLA

Eindhoven University of Technology, Eindhoven, The Netherlands and University of Kansas, Lawrence, Kansas

AND

STeven Van de Par

University of Oldenburg, Oldenburg, Germany

\begin{abstract}
Synchrony perception for audio-visual stimulus pairs is typically studied by using temporal order judgment (TOJ) or synchrony judgment (SJ) tasks. Research has shown that estimates of the point of subjective simultaneity (PSS) obtained using these two methods do not necessarily correspond. Here, we investigate the hypothesis that the PSS estimate obtained in a TOJ task is shifted in the direction of the most sensitive part of the synchrony judgment curve, as obtained in an SJ task. The results confirm that criterion shifts in the TOJ task move the PSS toward regions of the audio-visual temporal interval continuum where discriminations are most sensitive.
\end{abstract}

Seeing and hearing a hammer hitting a peg, a ball bouncing on the street, or a person clapping his or her hands are all examples of simple audio-visual events. In nature, the relative delay between the arrival of auditory and visual components of an event at an observer's eyes and ears is determined by the distance from the event to the observer and the relative propagation times of sound and light. In the laboratory, it is possible to decouple the auditory and visual components of a single event to various degrees of physical asynchrony. These are scaled along an audio-visual temporal interval continuum in which the point of objective synchrony (POS) is set equal to $0 \mathrm{msec}$, negative values conventionally refer to auditory-leading pairs, and positive values refer to visual-leading pairs (see, e.g., Arrighi, Alais, \& Burr, 2006; Aschersleben \& Müsseler, 1999; Enoki, Washikita, \& Yamada, 2006; Vatakis \& Spence, 2006a, 2006b; Zampini, Shore, \& Spence, 2003a, 2003b; but for exceptions, see Lewald \& Guski, 2004; Spence, Baddeley, Zampini, James, \& Shore, 2003). Subjective audio-visual simultaneity can be assessed by presenting observers with audio-visual temporal intervals, which are judged using a temporal order judgment (TOJ) task or a synchrony judgment (SJ) task. In a TOJ task, observers judge whether the auditory or the visual event comes first. The TOJ task can be extended by adding synchronous as a third response option (synchrony judgment with three response options; SJ3 task). Alternatively, a synchrony judgment task can also be limited to synchronous and asynchronous response options (SJ2).

In a previous study (van Eijk, Kohlrausch, Juola, \& van de Par, 2008), we have shown that estimates of the point of subjective simultaneity (PSS) obtained from a TOJ task were uncorrelated with PSS estimates obtained from SJ2 and SJ3 tasks, whereas the PSS values obtained from the two SJ tasks did correlate.

The deviant PSS estimates obtained from TOJ data might be explained by differences in sensitivity to audiovisual temporal intervals along the audio-visual temporal interval continuum (van Eijk, Kohlrausch, Juola, \& van de Par, 2009). In the study by van Eijk et al. (2008), two types of audio-visual stimulus pairs were used: a continuous event consisting of a bouncing ball, which appeared to drop down to a surface and rise up again, accompanied by an appropriate impact sound, and an isolated flash and click without any contextual event. The slopes of the synchronous response curves at the transition from perceived synchrony to perceived asynchrony (called synchrony boundaries) were steeper at the auditory-leading synchrony boundary than at the visual-leading synchrony boundary when the bouncing ball stimulus was used. The

R. L. J.van Eijk, r.l.j.v.eijk@tue.nl 
PSS estimate obtained from TOJ data $(7 \mathrm{msec})$ was located nearer the auditory-leading synchrony boundary, as compared with the PSS estimate obtained from SJ data $(37 \mathrm{msec})$; that is, the TOJ PSS estimate was shifted in the direction of the steeper of the two slopes derived from SJs. For the flash-click stimulus, however, no sensitivity difference at the two synchrony boundaries was found. Correspondingly, the PSS estimates obtained from TOJ data $(21 \mathrm{msec})$ and SJ data $(22 \mathrm{msec})$ also did not differ, providing further support for an effect of sensitivity differences along the audio-visual temporal interval continuum on the resulting PSS value estimated from TOJ data.

In the present study, we tested the hypothesis that individual PSS estimates from TOJ data are influenced by the relative slope steepness at the synchrony boundaries estimated in an SJ task. In order to test this hypothesis within the context of a single underlying audio-visual event, a stimulus is required that can be manipulated in such a way that the relation between the slopes on the two sides of the synchrony range is systematically altered. Ideally, such a stimulus should have a steeper slope on the auditoryleading side of the temporal continuum for one condition and a steeper slope on the visual-leading side of the temporal continuum for another condition (i.e., a reversal of slope asymmetry between two conditions). If our hypothesis holds true, such a reversal in slope asymmetry should evoke a clear corresponding shift in TOJ PSS.

A potential stimulus manipulation for achieving different slope asymmetries for different stimulus conditions is to vary the availability of visual predictive information. In the study by van Eijk et al. (2008), the slopes of the synchronous response curves at the synchrony boundaries were steeper at the auditory-leading synchrony boundary than at the visual-leading synchrony boundary when visual predictive and postdictive information was available (in the bouncing ball stimulus). A stimulus with visual predictive information thus results in greater sensitivity at the transition from perceived synchrony to perceived asynchrony for auditory-leading asynchronies than for visual-leading ones. No asymmetry in sensitivity at the synchrony boundaries was found, however, when neither visual predictive nor postdictive information was present in the (flash-click) stimulus. These findings suggest that manipulating the availability of visual predictive information allows us to vary the slope asymmetry present in SJ data. Furthermore, we expect that manipulation of the availability of visual postdictive information will result in a steeper slope at the visual-leading synchrony boundary, analogously (but opposite) to the manipulation of visual predictive information. That is, when visual postdictive information (in the absence of predictive information) provides feedback that allows a participant to determine the approximate (past) moment of occurrence of a visual event, higher sensitivity can be expected for the visual-leading (or auditory-lagging) temporal intervals than when no such information is present.

A requirement for testing our hypothesis was the use of a single audio-visual stimulus with a common (simulated) underlying physical event that allowed independent manipulation of the availability of visual predictive and postdictive information. This requirement can be realized with a visual stimulus simulating a Newton's cradle toy: five balls suspended from strings, with the leftmost ball initially describing a left-to-right pendulum movement, impacting its neighbor and, as a result, launching the rightmost ball (see Figure 1). In a physically synchronous presentation, an impact sound is produced at the moment of visual (physical) impact. The spatial separation between the initial leftmost swing and the resulting rightmost swing allows independent manipulation of visual predictive and postdictive information. That is, visual predictive information was manipulated by either showing or hiding the initial leftmost swing of the pendulum movement, whereas visual postdictive information was manipulated by either showing or hiding the following rightmost swing. The SJ task was used in order to verify whether these stimulus manipulations resulted in the expected reversal in slope asymmetry, whereas the TOJ task was used to obtain PSS estimates that could be related to any slope asymmetry found in the SJ task.

\section{METHOD}

In the present study, the perceived audio-visual temporal relationship was measured for three visual conditions of the simulated Newton's cradle toy, using two experimental methods. Two of the three visual conditions always showed the left half of the stimulus and, thus, contained the visual predictive information available in the left-to-right movement of the leftmost ball: In the entire condition, the entire Newton's cradle toy was visible, whereas in the left-half condition, only the left half of the stimulus was visible, whereas the right half of the stimulus was covered by an occluding panel. As a result, the launching of the rightmost ball was hidden from view in the left-half condition, making visual postdictive information unavailable to the observer. In the right-half condition, the launching of the rightmost ball was visible, but the left half of the stimulus was occluded, hiding the initial predictive movement of the leftmost ball.

The auditory component of the stimulus consisted of a synthesized impact sound realistically simulating the sound produced by two metal balls striking each other. Stimuli were presented with a relative audio-visual delay in the range of $-300 \mathrm{msec}$ (audio leading) to $+300 \mathrm{msec}$ (video leading) in steps of $50 \mathrm{msec}$, with the addition of -25 -msec and +25 -msec delays. After each single stimulus presentation, the participants either indicated the temporal order of the two events (TOJ task) or judged synchrony or successiveness, using an SJ task with three response categories: audio first, synchronous, or video first (also called ternary TOJ; Ulrich, 1987). The experimental method is discussed in more detail below.

\section{Participants}

Five female and 11 male participants took part, including the authors. Three participants were paid, whereas the others participated voluntarily. The participants varied in age from 23 to 60 years, with a mean of 33. All the participants reported normal or corrected-tonormal vision and normal hearing ability.

\section{Stimulus}

The Newton's cradle toy simulation consisted of five white disks (or "balls," $94 \mathrm{~cd} / \mathrm{m}^{2}$, as measured using an LMT L1003 luminance meter) against a black background. The disks had a diameter of 49 pixels and subtended an area of $1.4^{\circ}$ at an unconstrained viewing distance of about $60 \mathrm{~cm}$. Each disk appeared to be suspended from a rigid white string ( 3 pixels wide $\times 300$ pixels long, measured from the middle of the ball) that was attached to a white bar (220 pixels wide $\times 10$ pixels high). Initially, the four rightmost balls appeared to be suspended from strings with a vertical orientation, whereas the remaining (leftmost) ball was "lifted" from its stationary position in such a way that its string was oriented horizontally (see the first 

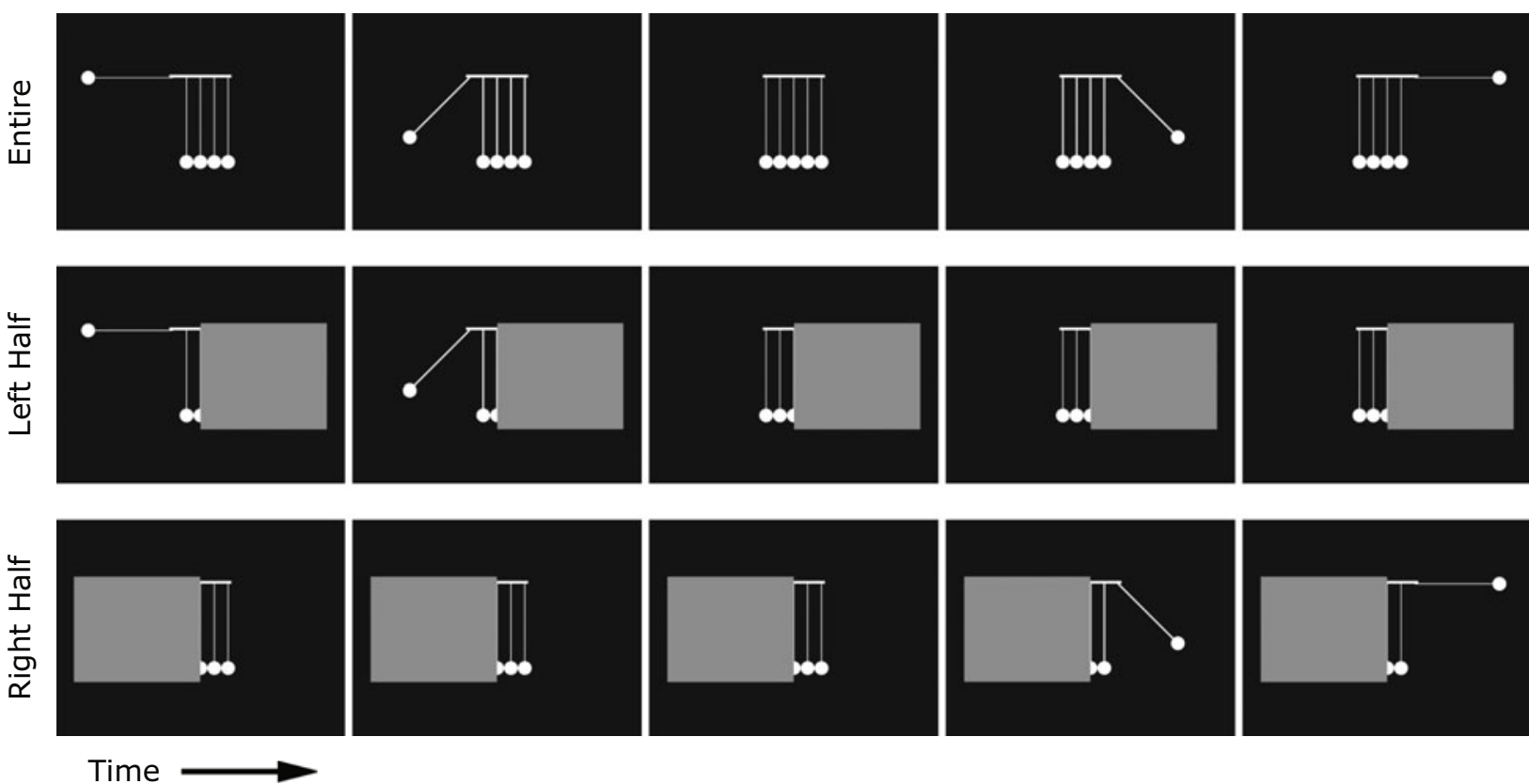

Figure 1. The three visual conditions of the Newton's cradle toy simulation, shown at five different moments in time. The top row shows the entire conditions, the middle row shows the left-half condition, and the bottom row shows the right-half condition. The first column shows the stimulus before motion onset, the third column shows the stimulus at the moment of visual impact, and the fifth column shows the stimulus after the motion has ended. The second and fourth columns show intermediate positions. Note that the five columns do not represent the stimulus at equal time intervals.

column of Figure 1). The animation consisted of the left ball apparently swinging toward its neighboring ball, hitting it, and thus causing the rightmost ball to be launched (columns two through four of Figure 1). The animation ended when the rightmost ball had reached the position in which its string was oriented horizontally (column five of Figure 1).

The movement of the ball was modeled according to a physically plausible model of pendulum movement. Before the onset of the movement of the leftmost ball, it remained "floating" at its initial position for a random period within the range of $47-447 \mathrm{msec}$. The left-to-right pendulum movement, jointly described by the leftmost and rightmost balls, had a constant duration of $1,317 \mathrm{msec}$, with the visual impact of the leftmost ball (and thus the launching of the rightmost ball) occurring halfway - that is, $659 \mathrm{msec}$ after onset of the movement. The motion pattern of the balls was identical for each stimulus presentation, but the onset of the movement was randomized such that the visual impact occurred at a random moment after the start of the stimulus presentation in the range of approximately $900 \pm 200 \mathrm{msec}$ (with the restriction that the moment of impact was actually visible in one frame).

The three visual stimulus conditions were created by showing the entire Newton's cradle toy or by hiding either the left or the right half of the toy by superimposing a gray plane $\left(22 \mathrm{~cd} / \mathrm{m}^{2}\right)$ in front of the respective half. The acoustic part of the stimulus consisted of a short sound that could be interpreted as being produced by two metal balls hitting each other. The sound consisted of 25 spectral components with an instantaneous onset and an exponentially decaying envelope $\mathrm{e}^{-t / \mathrm{T}}$, with $\mathrm{T}=3 \mathrm{msec}$. The constituent frequency components initially were in the range from 3000 to $12000 \mathrm{~Hz}$ at equal logarithmic intervals but were linearly decreased by $3000 \mathrm{~Hz}$ over a period of $80 \mathrm{msec}$. At the moment of onset, the auditory stimulus had a sound pressure level of $105 \mathrm{~dB}$.

\section{Apparatus}

The animation was shown on a Dell D1025HE CRT monitor at a resolution of $1,024 \times 768$ pixels and at an $85-\mathrm{Hz}$ refresh rate. Audio was played through a Creative SB Live! sound card, a Fostex PH-50 headphone amplifier, and Sennheiser HD 265 linear headphones. The participants were seated in front of the monitor and responded using a keyboard. The setting was a dimly lit sound-attenuated room.

The timing control of the auditory and visual signals was accurate to within $\pm 2 \mathrm{msec}$ as determined during a calibration procedure by using a photocell that measured the light emitted by a flashing disk on the screen. The electrical output of the photocell was shown as a trace on an oscilloscope together with a pulsed tone that was played in synchrony with the flashing disk on the screen.

\section{Design}

Three parameters were systematically manipulated: (1) visual stimulus (three values: entire, left half, and right half), (2) audio delay (15 values from $-300 \mathrm{msec}$ [auditory first] to $+300 \mathrm{msec}$ [visual first] in steps of $50 \mathrm{msec}$ plus $-25 \mathrm{msec}$ and $+25 \mathrm{msec}$ ), and (3) response rule (two values: TOJ and SJ). This resulted in a total of 90 conditions.

Audio-visual delays were selected to include the range where PSS estimates obtained from both TOJ and SJ tasks are generally observed (i.e., approximately between -100 and $+100 \mathrm{msec}$; see van Eijk et al., 2008, for a review). The smallest and largest delays were selected such that they could reasonably be expected to result in perfect observer performance. That is, those delays are generally large enough to determine whether the event in the auditory or visual modality had been presented first, resulting in a clearly asynchronous event. Since asynchronous responses have generally reached their minimum proportion at \pm 250 -msec delays (van Eijk et al., 2008), it was decided to limit the range of audio-visual delays to $\pm 300 \mathrm{msec}$. Finally, audio-visual delays were centered around $0 \mathrm{msec}$ in order to prevent temporal recalibration to a nonzero delay (see, e.g., Fujisaki, Shimojo, Kashino, \& Nishida, 2004; Navarra, et al., 2005; Vatakis, Navarra, Soto-Faraco, \& Spence, 2008; Vroomen, Keetels, de Gelder, \& Bertelson, 2004).

The experiment was divided into eight sessions. In the first four sessions, one of the response rules (TOJ or SJ) was used, and in the last four sessions, the other rule was used. The order in which the response rules were used was counterbalanced across participants. 
Each session always started with the entire condition. The order of the subsequent left-half and right-half conditions was reversed from session to session and counterbalanced both between and within participants. All audio delay conditions were presented 15 times in each session for each of the three visual stimulus conditions, resulting in 60 measurements for each audio delay in each of the six visual stimulus $\times$ response rule combinations.

\section{Procedure}

Prior to the start of the experiment, the participants were informed about the content of the audio-visual animation via written instructions. In the TOJ condition, the participants were asked to use the keyboard to "indicate whether the sound preceded the visual collision (' 1 ' key) or whether the visual collision preceded the sound (' 2 ')." In the SJ condition, the participants were asked to "indicate whether the sound preceded the visual collision (' 1 ' key), whether the sound occurred simultaneously with the visual collision ('2') or whether the visual collision preceded the sound (' 3 ')." The response was briefly indicated on the screen. After reading the instructions, the participants were allowed to ask questions, after which the experiment started with the first practice phase. During the first practice phase, the experimenter was present to make sure everything was clear. Each experimental session was divided into three main parts, with each main part following the same structure: a practice phase consisting of at least 15 stimuli containing all audio delays, followed by three measurement phases of 75 stimuli each (i.e., five presentations of each audio delay in a random order). The participants were allowed to take a break between phases. In order to prime the participants with the full physical event underlying the left-half and right-half conditions, the first main part of each session always contained the entire condition, whereas the next two main parts contained the left-half and right-half conditions in counterbalanced order. Each session lasted for 30-45 min, except for the first one, which lasted for about 50-55 min due to longer training sessions ( 45 stimuli, instead of 15 for each practice phase in most of the later sessions). The first practice phase of the fifth session consisted of 30 instead of 15 stimuli, in order to allow the participants more time to adjust to the new response rule.

\section{RESULTS}

The results for the SJ task are treated first in order to ascertain whether the stimulus manipulations resulted in different slope asymmetries, as expressed in the introduc- tion (i.e., a steeper slope at the auditory-leading synchrony boundary when visual predictive information is available, and a steeper slope at the visual-leading synchrony boundary when visual postdictive information is present but visual predictive information is not available). Second, the results of the TOJ task are related to the slope asymmetry data from the SJ task. Two participants were removed from the analysis due to reasons explained below.

\section{SJ Data}

Raw data averaged over the 14 remaining participants are shown in the left panel of Figure 2, which shows the proportion of synchronous responses as a function of relative delay for all three visual conditions (audio-first and video-first response curves are left out for clarity reasons). From Figure 2, it is apparent that entire and left-half conditions produced very similar synchronous response curves, whereas the right-half visual condition resulted in a clear shift in the SJ data toward more negative, auditory-leading delays, as compared with the entire and left-half conditions.

The SJ data for each individual participant were fitted by cumulative Gaussian functions for each of the three response categories (i.e., audio first, synchronous, and video first). In order to allow for different slopes in the two halves of the synchrony curve, we used two cumulative Gaussians, instead of one normal distribution, to fit the overall synchrony response curve (similar to the procedure in Smeele, 1994, and identical to that in van Eijk et al., 2008). The parameters resulting from the fitting of a cumulative Gaussian distribution to response data can be influenced by stimulus-independent lapses (e.g., pressing the wrong key, blinking during stimulus presentation). Therefore, the data were fitted using the psignifit toolbox, Version 2.5.6, for MATLAB (see http:// bootstrap-software.org/psignifit/), which implements the maximum-likelihood method described by Wichmann and Hill (2001). This method fits the data with a psychometric function of the form $\gamma+(1-\gamma-\lambda) F(\alpha, \beta)$, with
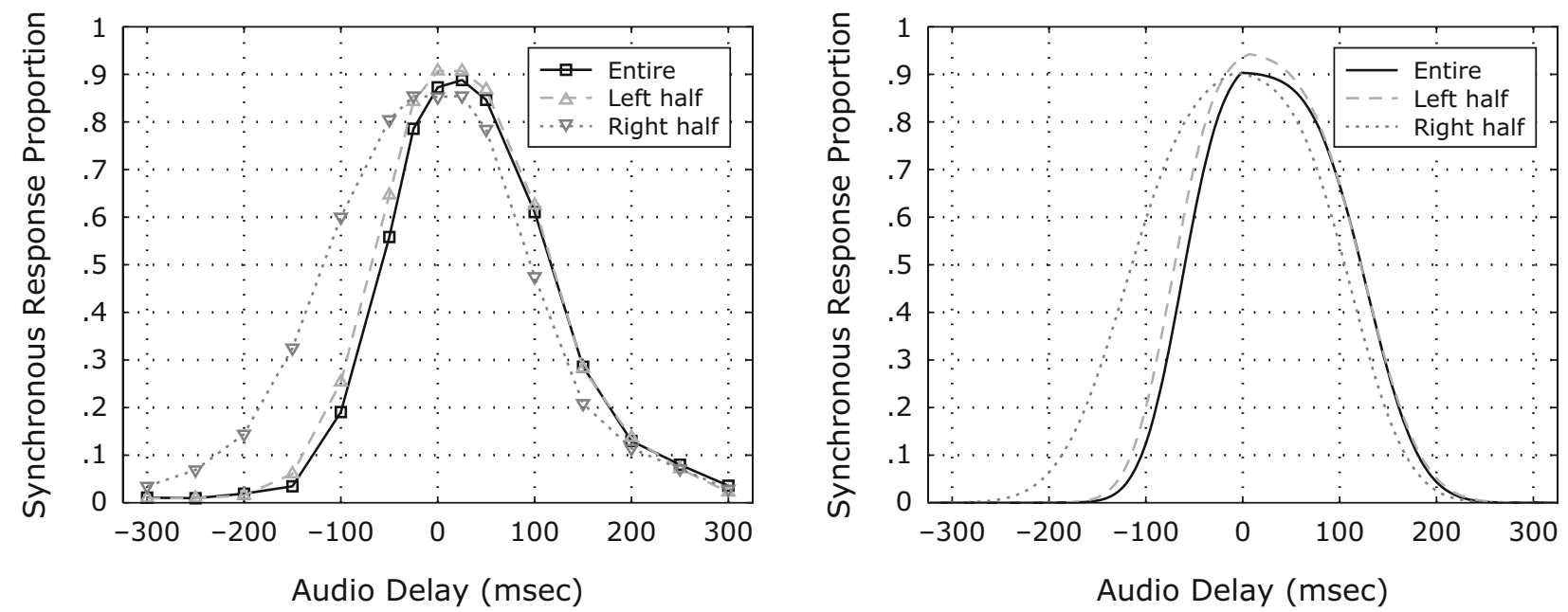

Figure 2. Raw and fitted synchronous responses for all three visual conditions, averaged over participants $(N=14)$. The left panel shows average synchronous response proportions as a function of the relative delay. The right panel shows fitted synchrony curves determined by averaging individually determined fitting parameters over participants. 
$F$ being the cumulative Gaussian distribution with mean $\alpha$ and standard deviation $\beta$. For synchronous response data, lapses were fitted by using $\gamma$ (lower horizontal asymptote), whereas the $\lambda$ parameter fits the maximum proportion of synchronous responses. After fitting, the $\gamma$ parameter was removed from the theoretical, or underlying, psychometric function, which is assumed to represent the actual perception of the participant (rather than observed performance). Audio-first and video-first data were fitted with the same psychometric function, but since raw data proportions by definition add up to one (i.e., the data are complementary), only the $\lambda$ parameter was removed (i.e., set to zero) in order to maintain the complementary aspect of the responses in the data fits. Average synchronous response curves (determined by averaging the fitting parameters of individual synchronous curves) are plotted in the right panel of Figure 2 for all three visual conditions.

After fitting the individual response data, slope asymmetries were determined. Two steps are involved in calculating slope asymmetries: (1) determining synchrony boundaries, and (2) determining the slope at each synchrony boundary. Synchrony boundaries were determined by calculating the intersection points of the (fitted) synchrony curves with their neighboring audio-first and video-first response curves. Then the slopes of the synchrony curves were determined at the two synchrony boundaries. Two participants were removed from further analysis, since their synchronous responses were relatively constant over the range of relative delays for either one or all three of the visual conditions. As a result, no synchrony boundaries and, thus, no slopes could be calculated. Average slopes are shown in Table 1.

It can be seen in Table 1 and Figure 2 that the slopes on either side of the synchrony curves are not the same across conditions. A 3 (visual condition) $\times 2$ (location; i.e., audio first or video first) repeated measures ANOVA indeed yielded a significant visual condition $\times$ location interaction in the slope data $[F(1.26,16.44)=4.69, p=.038$,

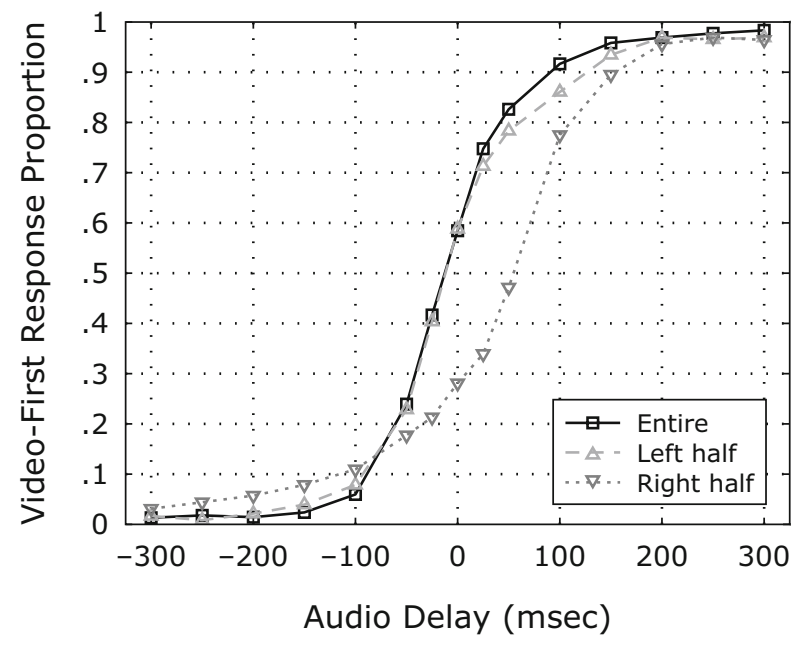

Table 1

Average Slopes at Audio-First and Video-First Synchrony Boundaries for Synchrony Judgment Data and Average Point of Subjective Simultaneity (PSS) Values for Temporal Order Judgment (TOJ) Data for All Three Visual Conditions of the Newton's Cradle Toy Stimulus, Derived From Fitted Curves

\begin{tabular}{|c|c|c|c|c|c|c|}
\hline \multirow[b]{3}{*}{ Condition } & \multicolumn{4}{|c|}{ Slope $\left(\times 10^{-2} \delta\right.$ Proportion $\left./ \mathrm{msec}\right)$} & \multirow{2}{*}{\multicolumn{2}{|c|}{$\begin{array}{c}\text { TOJ PSS } \\
(\mathrm{msec})\end{array}$}} \\
\hline & \multicolumn{2}{|c|}{ Audio First } & \multicolumn{2}{|c|}{ Video First } & & \\
\hline & $M$ & $S E$ & $M$ & $S E$ & $M$ & $S E$ \\
\hline Entire & 1.21 & 0.11 & 0.88 & 0.10 & -8 & 7 \\
\hline Left half & 1.21 & 0.10 & 0.95 & 0.10 & -5 & 7 \\
\hline Right half & 0.76 & 0.10 & 0.87 & 0.10 & 50 & 9 \\
\hline
\end{tabular}

$\varepsilon=0.63] .{ }^{1}$ Specifically, the audio-first slope was steeper than the video-first slope for both entire $\left[1.21 \times 10^{-2}\right.$ vs. $0.88 \times 10^{-2} \delta$ proportion $\left./ \mathrm{msec} ; t(13)=2.69, p=.019\right]$ and left-half $\left[1.21 \times 10^{-2}\right.$ vs. $0.95 \times 10^{-2} \delta$ proportion/ msec; $t(13)=1.98, p=.069$; i.e., only marginally significant] conditions. ${ }^{2}$ However, for the right-half condition, there was a nonsignificant trend in the opposite direction $\left[0.76 \times 10^{-2}\right.$ vs. $0.87 \times 10^{-2} \delta$ proportion $/ \mathrm{msec}$; $t(13)<1]$. It can thus be concluded that, on average, the audio-first synchrony boundary tends to have a steeper slope than does the video-first synchrony boundary for entire and left-half conditions, but that no significant slope asymmetry is present in the right-half condition. More importantly, the significant interaction between visual condition and location demonstrates that slope asymmetries vary with visual condition. Whether PSS estimates obtained from TOJ data indeed are related to the slope asymmetry from SJ data is analyzed in the next section.

\section{TOJ Data}

Raw data averaged over all participants are shown in the left panel of Figure 3, which shows the proportion of video-first responses as a function of relative delay for all three visual conditions (audio-first response curves are left out for reasons of clarity). From Figure 3, it is

Figure 3. Raw and fitted video-first responses for all three visual conditions averaged over participants $(N=14)$. The left panel shows average video-first response proportions as a function of the relative delay. The right panel shows fitted video-first curves determined by averaging individually determined fitting parameters over participants. 
clear that the entire and left-half conditions produced very similar response patterns, whereas the right-half visual condition resulted in a clear shift in the direction of more positive delays (as compared with the entire and left-half conditions).

Video-first responses of individual participants were fitted using the same psychometric function as those used for fitting the SJ data. Unlike the synchronous response data, TOJ performance can be expected to be perfect (i.e., 0.0 and 1.0 response proportions) at relative delays of $\pm 250 \mathrm{msec}$ or more. Therefore, for TOJ data, both $\gamma$ and $\lambda$ parameters are removed (i.e., set to zero) from the fitted psychometric function to arrive at the theoretical, or underlying, psychometric function. Average video-first response curves (determined by averaging the fitting parameters of individual video-first curves) are plotted in the right panel of Figure 3 for all three visual conditions. For TOJ data, the PSS is defined as the TOJ $50 \%$ point, or the mean of the cumulative Gaussian distribution fitted to the raw data. Average PSS values are shown in Table 1.

Our hypothesis states that individual PSS estimates from TOJ data are influenced by the relative slope steepness at the synchrony boundaries estimated in an SJ task. The average PSS values for SJ and TOJ are shown in Figure 4, which plots average response curves for SJ and TOJ for the same visual conditions in separate panels. Average response curves were determined by averaging the fitting parameters of individual synchronous and video-first response curves. As can be seen in Figure 4, the average TOJ PSS values for the entire and left-half visual conditions are between the audio-first synchrony boundary and the PSS - that is, closer to the steeper audio-first synchrony boundary than to the video-first synchrony boundary. However, the average TOJ PSS for the right-half condition is closer to the video-first synchrony boundary, which is steeper (n.s.) on average than the audio-first synchrony boundary for the right-half condition. In general, TOJ PSS values tend to be closer to the steeper synchrony boundary - that is, the location where temporal interval discrimination sensitivity is higher. Although this observation already lends some support to the hypothesis that TOJ PSS estimates are influenced by the relative steepness of SJ slopes, it was tested more critically by relating the (position of) individual TOJ PSS estimates to the relative steepness of audio-first and video-first slopes for each participant.

In order to relate the (position of the) TOJ PSS to the relative steepness of audio-first and video-first slopes, both the TOJ PSS and the ratios of the slopes at the synchrony boundaries were normalized. The TOJ PSS was normalized to reflect its relative position with respect to the synchrony boundaries in such a way that the audiofirst and video-first synchrony boundaries corresponded to normalized values of -1 and +1 , respectively, and the PSS to a normalized value of 0 . Slope normalization was done by taking the $\log _{2}$ of the ratio of the video-first slope to the audio-first slope. Using the ratio of slopes normalizes for differences in absolute slope, whereas the logarithm has the advantage that two slope ratios consisting of identical slopes, but reversed for audio-first and video-

\section{Entire Condition}
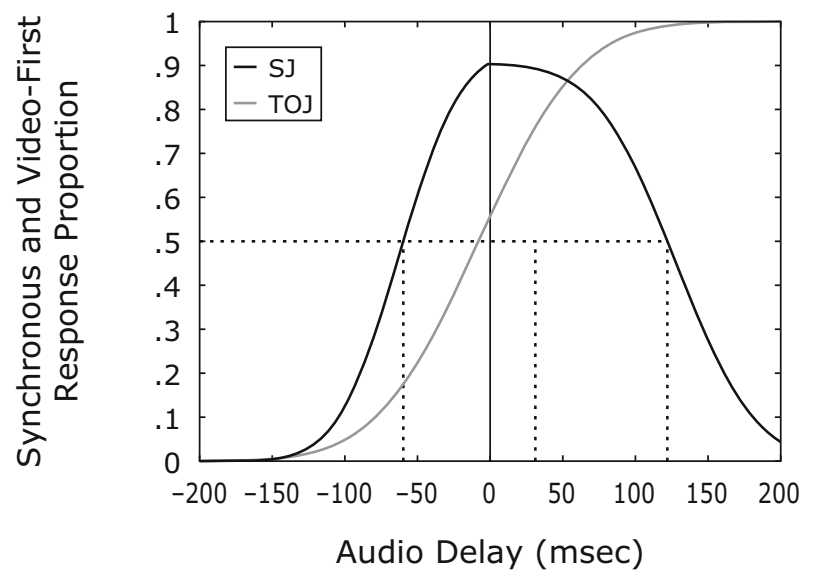

Left-Half Condition
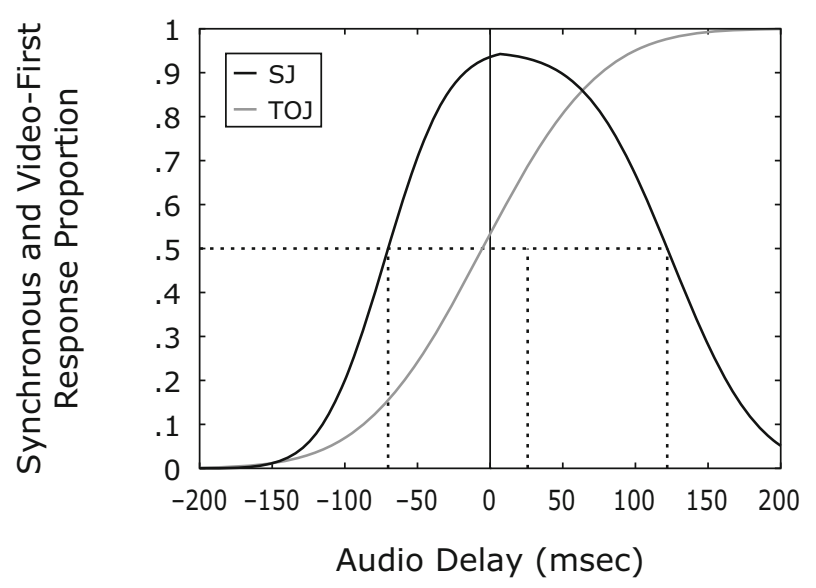

Right-Half Condition
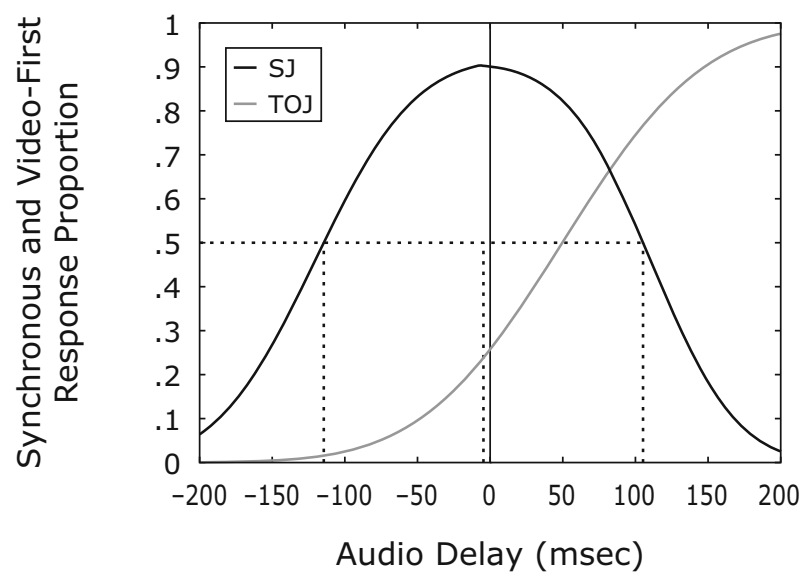

Figure 4. Fitted synchronous and video-first responses for all three visual conditions, averaged over participants $(N=14)$. The upper panel shows the entire condition, the middle panel shows the left-half condition, and the lower panel shows the right-half condition. Vertical lines indicate audio-first and video-first synchrony boundaries and the point of subjective simultaneity (PSS). Average temporal order judgment (TOJ) PSS values (50\% points) are closer to the audio-first synchrony boundary for the entire and left-half conditions but are closer to the video-first synchrony boundary for the right-half condition. 


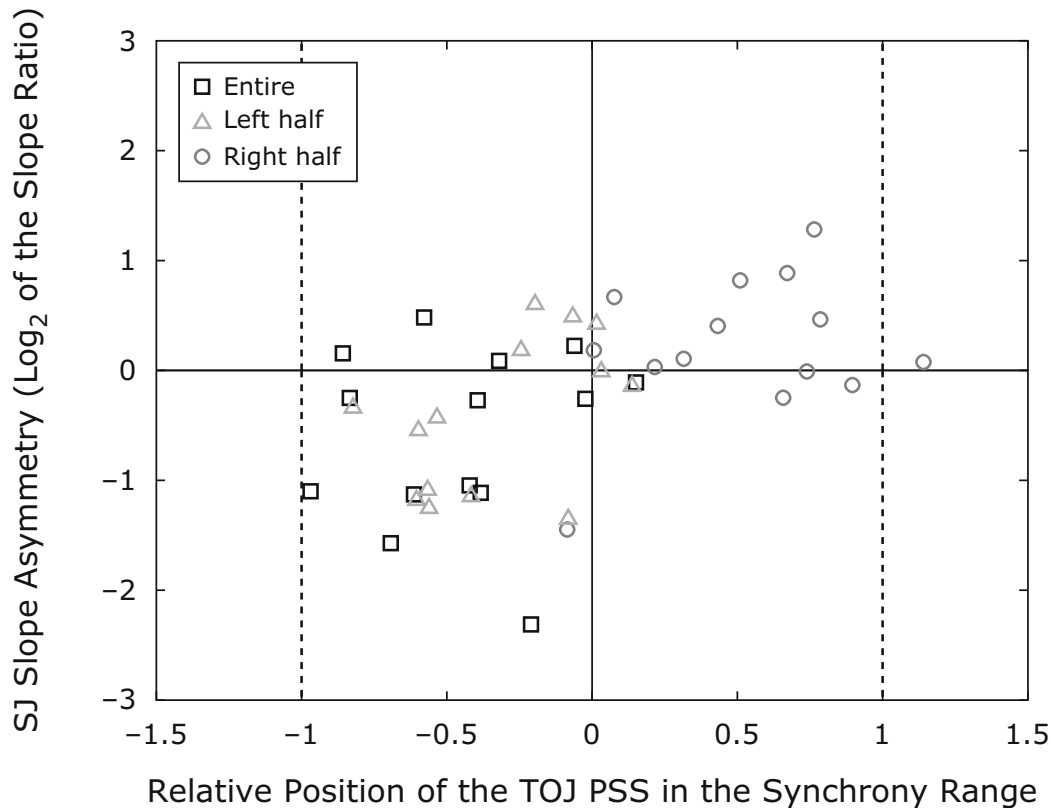

Figure 5. Log slope ratios of slopes measured at synchrony judgment (SJ) synchrony boundaries plotted against individually normalized temporal order judgment (TOJ) point of subjective simultaneity (PSS) values for all three conditions.

first synchrony boundaries (e.g., $\frac{2}{1}$ and $\frac{1}{2}$ ), will yield the same asymmetry measure but with opposite signs (e.g., +1 , and -1$)$. Negative log slope ratios indicate steeper slopes (i.e., higher sensitivity) at the audio-first synchrony boundary, whereas positive log slope ratios indicate steeper slopes at the video-first synchrony boundary. In Figure 5, the relationship is plotted between the individually determined relative position of the TOJ PSS (relative to the synchrony boundaries) and the corresponding relative steepness of the slopes at the synchrony boundaries. It can be seen from Figure 5 that virtually all TOJ PSS estimates are within the synchrony range - that is, within the -1 to +1 range (see also van Eijk et al., 2008, for a similar observation).

From Figure 5, it can be seen that there is a positive relation between the relative position of the TOJ PSS in the synchrony range and the log slope ratio: Participants with a negative log slope ratio (i.e., higher sensitivity at the audio-first synchrony boundary) have their TOJ PSS values closer to the audio-first synchrony boundary, whereas participants with a positive log slope ratio (i.e., higher sensitivity at the video-first synchrony boundary) have their TOJ PSS values closer to the video-first synchrony boundary. The relationship between relative TOJ PSS location and slope log ratio is significant $(r=.52, p<.001$, $N=42$ ), thus providing support for our hypothesis that TOJ PSS estimates are shifted in the direction of the synchrony boundary that has the steeper slope. ${ }^{3}$

\section{DISCUSSION}

The main goal of the present research was to test the hypothesis that TOJs are influenced by the relative ease of detecting auditory-leading and visual-leading compo- nents of a bimodal stimulus. In the present study, the PSS estimated from the TOJ data was shifted in the direction of the most sensitive part of the SJ curve, as determined in an SJ task using the same stimuli. We demonstrated that the TOJ 50\% points (PSS values) tend to move toward the part of the audio-visual relative-delay continuum where audio-first or video-first synchrony judgments change more steeply to synchronous judgments. Within individual participants' data, the larger the (relative) difference in steepness of the two sides of the synchrony response category, the more pronounced is the shift in TOJ PSS values toward the synchrony boundary where sensitivity to audio-visual delays is higher (see Figure 5). When the data were averaged across participants, the slopes at the audio-first synchrony boundary were steeper than the slopes at the video-first boundary, but only when the left half of the stimulus was visible (i.e., for entire and left-half conditions; see Figure 1). A reversed trend was observed for the right-half visual condition: Audio-first slopes were flatter (n.s.) than video-first slopes. This observation suggests that performance on TOJ tasks is influenced by a response strategy that puts the decision criterion close to the point along the audio-visual delay continuum where observer sensitivity is highest.

In order to test our hypothesis, a stimulus was needed with different slope asymmetries for different visual conditions. Others studies in which an SJ task has been used generally have not reported slopes of the synchronous response curve, however (see, e.g., Arrighi et al., 2006; Enoki et al., 2006; Fujisaki et al., 2004; Smeele, 1994; Stone et al., 2001; Vatakis et al., 2008; Zampini, Guest, Shore, \& Spence, 2005; but see van Eijk et al., 2008, for an exception). As a result of the absence of slope measures in the literature, we had to find a stimulus where 
the slope asymmetry could be varied by manipulating some stimulus property. It was found that manipulating the availability of visual predictive and postdictive information in a Newton's cradle stimulus resulted in steeper slopes at the auditory-leading synchrony boundaries when visual predictive information was available (i.e., in entire and left-half conditions), but that the slope asymmetry was reversed (n.s.) when only visual postdictive information was available (in the right-half condition). In addition to the presence or absence of predictive and postdictive information in the various visual conditions, it was noted that the phenomenological properties of the stimuli were also changed. That is, when the stimulus appeared to represent a ball swinging down and striking the stationary balls, the unavoidable conclusion in most audio-visual delay conditions was that the strike caused the sound. On the other hand, when the initial apparent movement was blocked by the gray panel, the unmistakable conclusion in most conditions was that the sound caused the apparent motion.

The influence of visual predictive information in speech on the subsequent processing of the auditory speech signal was investigated in a study by van Wassenhove, Grant, and Poeppel (2005). They combined EEG measurements with a psychophysical experiment in which they asked participants to judge whether an audio-visual (McGurk or congruent) speech utterance was either "ka," "pa," or "ta." Their results indicated that auditory stimulus processing speeds up when visual speech correctly predicts the subsequent auditory speech. It is important to note that the task in van Wassenhove et al.'s study was speech identification, whereas the task in the present study was to indicate the perceived temporal relation between events in different modalities. Nevertheless, it is conceivable that speeding up of auditory processing also occurred in the present study when visual predictive information was available. This speeding up of auditory processing might have resulted in the steeper audio-first slopes observed for entire and left-half conditions. Besides acting as a predictor of auditory speech, the visual component of audio-visual speech also acts as the cause of auditory speech. Therefore, the results of van Wassenhove et al. cannot disambiguate between the effect of visual predictive information and apparent causality.

Whether the observed change in slope asymmetry is due to the manipulation of visual predictive and postdictive information or apparent causal interactions thus remains to be determined. Although the potential effect of the causal interpretation on perceived simultaneity is an intriguing idea, it remains a subject for future research to disentangle the effects of visual predictive information and apparent causality. Potential avenues to take include using a stimulus with auditory predictive information and using a stimulus of a ball bouncing off an invisible bar, since this does have a causal interpretation but offers limited visual predictive information. The relation between slope asymmetry and TOJ PSS estimates that was demonstrated here, however, remains unequivocally true regardless of the outcome of such experiments.
We believe that the present study is the first to demonstrate the relationship between the relative steepness of the synchrony boundaries and the subsequent location of TOJ PSS estimates. This relationship suggests that the TOJ response shifts observed in the present study resulted, at least in part, from strategic variations in criteria toward the audio-visual delay region of greater observer sensitivity, where such judgments are easier to make.

\section{AUTHOR NOTE}

Thanks to Martin Boschman, Constant Hak, Dik Hermes, and Christophe Stoelinga for technical assistance. We also acknowledge support from a grant from NWO (Nederlandse Organisatie voor Wetenschappelijk Onderzoek; Dutch Organization for Scientific Research) to the third author. Thanks are also due Lynne Nygaard and two anonymous reviewers for helpful comments on previous versions of the manuscript. Correspondence concerning this article should be addressed to R. L. J. van Eijk, Den Dolech 2, IPO 0.22, P.O. Box 513, 5600 MB Eindhoven, The Netherlands (r.1.j.v.eijk@tue.nl).

\section{REFERENCES}

Arrighi, R., Alais, D., \& Burr, D. (2006). Perceptual synchrony of audiovisual streams for natural and artificial motion sequences. Journal of Vision, 6, 260-268. doi:10.1167/6.3.6

Aschersleben, G., \& Müsseler, J. (1999). Dissociations in the timing of stationary and moving stimuli. Journal of Experimental Psychology: Human Perception \& Performance, 25, 1709-1720.

EnOKI, K., Washikita, K., \& YAmada, M. (2006). Detection threshold of asynchrony between auditory and visual stimuli for various motion patterns of a ball. In Proceedings of the 9th Western Pacific Acoustic Conference (WESPAC IX) [CD-ROM]. Seoul: Acoustical Society of Korea.

Fujisaki, W., Shimojo, S., Kashino, M., \& Nishida, S. (2004). Recalibration of audiovisual simultaneity. Nature Neuroscience, 7, 773-778. doi: $10.1038 / \mathrm{nn} 1268$

LEWALD, J., \& GUSKI, R. (2004). Auditory-visual temporal integration as a function of distance: No compensation for sound-transmission time in human perception. Neuroscience Letters, 357, 119-122. doi:10.1016/j.neulet.2003.12.045

Navarra, J., Vatakis, A., Zampini, M., Soto-Faraco, S., HumPHREYS, W., \& SPENCE, C. (2005). Exposure to asynchronous audiovisual speech extends the temporal window for audiovisual integration. Cognitive Brain Research, 25, 499-507. doi:10.1016/j.cogbrainres 2005.07.009

SMEELE, P. M. T. (1994). Perceiving speech: Integrating auditory and visual speech. Unpublished doctoral thesis, Technische Universiteit Delft, Delft, The Netherlands. Retrieved April 11, 2007, from http:// repository.tudelft.nl/file/257315/201176

Spence, C., Baddeley, R., Zampini, M., James, R., \& Shore, D. I. (2003). Multisensory temporal order judgments: When two locations are better than one. Perception \& Psychophysics, 65, 318-328.

Stone, J. V., Hunkin, N. M., Porrill, J., Wood, R., Keeler, V., BeanLAND, M., ET AL. (2001). When is now? Perception of simultaneity. Proceedings of the Royal Society of London B, 268, 31-38. doi:10.1098/ rspb. 2000.1326

ULRICH, R. (1987). Threshold models of temporal-order judgments evaluated by a ternary response task. Perception \& Psychophysics, 42, 224-239.

van Eijk, R. L. J., Kohlrausch, A., Juola, J. F., \& van de Par, S. (2008). Audiovisual synchrony and temporal order judgments: Effects of experimental method and stimulus type. Perception \& Psychophysics, 70, 955-968. doi:10.3758/PP.70.6.955

van Eijk, R. L. J., Kohlrausch, A., Juola, J. F., \& van de Par, S. (2009). Temporal interval discrimination thresholds depend on perceived synchrony for audio-visual stimulus pairs. Journal of Experimental Psychology: Human Perception \& Performance, 35, $1254-$ 1263. doi: $10.1037 / \mathrm{a} 0014254$

van Wassenhove, V., Grant, K. W., \& Poeppel, D. (2005). Visual speech speeds up the neural processing of auditory speech. Proceed- 
ings of the National Academy of Sciences, 102, 1181-1186. doi:10 .1073/pnas.0408949102

Vatakis, A., Navarra, J., Soto-Faraco, S., \& Spence, C. (2008). Audiovisual temporal adaptation of speech: Temporal order versus simultaneity judgments. Experimental Brain Research, 185, 521-529. doi:10.1007/s00221-007-1168-9

VATAKIS, A., \& SPEnce, C. (2006a). Audiovisual synchrony perception for music, speech, and object actions. Brain Research, 1111, 134-142. doi:10.1016/j.brainres.2006.05.078

VATAKIS, A., \& SPENCE, C. (2006b). Audiovisual synchrony perception for speech and music assessed using a temporal order judgment task. Neuroscience Letters, 393, 40-44. doi:10.1016/j.neulet.2005.09.032

Vroomen, J., Keetels, M., de Gelder, B., \& Bertelson, P. (2004). Recalibration of temporal order perception by exposure to audiovisual asynchrony. Cognitive Brain Research, 22, 32-35. doi:10.1016/ j.cogbrainres.2004.07.003

WichmanN, F. A., \& Hill, N. J. (2001). The psychometric function: I. Fitting, sampling, and goodness of fit. Perception \& Psychophysics, 63, 1293-1313.

Zampini, M., Guest, S., Shore, D. I., \& Spence, C. (2005). Audiovisual simultaneity judgments. Perception \& Psychophysics, 67, 531544.

Zampini, M., Shore, D. I., \& Spence, C. (2003a). Audiovisual temporal order judgments. Experimental Brain Research, 152, 198-210. doi:10.1007/s00221-003-1536-z

Zampini, M., Shore, D. I., \& Spence, C. (2003b). Multisensory temporal order judgments: The role of hemispheric redundancy. Interna- tional Journal of Psychophysiology, 50, 165-180. doi:10.1016/S0167 $-8760(03) 00132-6$

\section{NOTES}

1. All ANOVAs were performed using Greenhouse-Geisser adjusted numbers of degrees of freedom if the sphericity assumption was violated, as tested using Mauchley's test of sphericity. Only when a Greenhouse-Geisser adjustment of the degrees of freedom was applied is the correction factor $\varepsilon$ reported.

2. When visual predictive information is available - that is, in entire and left-half conditions - audio-first slopes are significantly steeper than video-first slopes $[F(1,13)=5.86, p=.031$, in a 2 (visual condition) $\times$ 2 (location) repeated measures ANOVA].

3. Repeating the correlation analysis without the authors left the relationship between relative TOJ PSS location and slope log ratio relatively unaffected $(r=.45, p=.014)$. Note that the authors were among the oldest participants in the experiment. That is, without the authors, the participants' ages varied from 23 to 45 years of age, with a mean of 29. Similar results are obtained when 2 additional participants ( 37 and 45 years of age $)$ are removed from the analysis $(r=.51, p=.010)$. A $2 \times 2$ chi-square test of contingency supported the overall correlation in Figure $5\left[\chi^{2}(1)=5.57 ; p=.018\right]$.

(Manuscript received February 24, 2009; revision accepted for publication June 20, 2010.) 